EPJ Web of Conferences 80, 00024 (2014)

DOI: $10.1051 /$ epj conf/ 20148000024

C) Owned by the authors, published by EDP Sciences, 2014

\title{
Precision QCD measurements at HERA
}

\author{
Hayk Pirumov ${ }^{1, \mathrm{a}}$ on behalf of $\mathrm{H} 1$ and ZEUS Collaborations \\ ${ }^{1}$ DESY, Notkestrasse 85, 22607 Hamburg, Germany
}

\begin{abstract}
A review of recent experimental results on perturbative QCD from the HERA experiments H1 and ZEUS is presented. All inclusive deep inelastic cross sections measured by the H1 and ZEUS collaborations in neutral and charged current unpolarised ep scattering are combined. They span six orders of magnitude in negative four-momentumtransfer squared, $Q^{2}$, and in Bjorken $x$. This data set is used as the sole input to NLO and NNLO QCD analyses to determine new sets of parton distributions, HERAPDF2.0, with small experimental uncertainties and an estimate of model and parametrisation uncertainties. Also shown are new results on inclusive jet, dijet and trijet differential cross sections measured in neutral current deep inelastic scattering. The precision jet data is used to extract the strong coupling $\alpha_{s}$ at NLO with small experimental errors.
\end{abstract}

\section{Introduction}

Deep-inelastic scattering (DIS) data provide high precision tests of perturbative quantum chromodynamics (QCD), and have led to a detailed and comprehensive understanding of proton structure.

The differential cross section for DIS ep scattering can be described in terms of proton structure functions $F_{2}, F_{L}$ and $x F_{3}$, which are related to parton distribution functions (PDFs). The structure functions depend on kinematic variables $x$ and $Q^{2}$ only, whereas the cross section is additionally dependent on the inelasticity $y$ related by $y=Q^{2} / s x$. The reduced neutral current differential cross section for $e^{+} p$ scattering can be written as

$$
\tilde{\sigma}_{N C}\left(x, Q^{2}, y\right) \equiv \frac{d^{2} \sigma_{N C}}{d x d Q^{2}} \frac{x Q^{4}}{2 \pi \alpha^{2}} \frac{1}{Y_{+}} \equiv\left(F_{2}-\frac{y^{2}}{Y_{+}} F_{L}-\frac{Y_{-}}{Y_{+}} x F_{3}\right),
$$

where $Y_{ \pm}=1 \pm(1-y)^{2}$ and the fine structure constant is defined as $\alpha \equiv \alpha\left(Q^{2}=0\right)$. As can be seen from the Eq. 1 the contribution of $F_{L}$ to the cross section is significant only at high $y$ and the contribution of the $x F_{3}$ is significant only for high values of $Q^{2}$. For the charged current (CC) processes the reduced differential cross section can be written in a form similar to Eq. 1 using the CC structure functions.

At the electron-proton collider HERA (1992-2007) electrons and positrons of $27.6 \mathrm{GeV}$ collided with protons of $920 \mathrm{GeV}$ corresponding to an $e p$ centre-of-mass energy of $319 \mathrm{GeV}$. The operation of HERA proceeded in two phases, HERA I (1992-2000) with an unpolarised and HERA II (2003-2007) with a longitudinally polarised lepton beam. At the end of the HERA data taking, special runs with reduced proton beam energies of 460 and $575 \mathrm{GeV}$ were taken for dedicated $F_{L}$ measurement. The two $e p$ interaction regions were instrumented with the multipurpose detectors of the H1 and ZEUS

ae-mail: hayk.pirumov@desy.de 
experiments. The inclusive NC and CC ep DIS cross section data obtained by the H1 and ZEUS experiments are used to determine the proton structure functions and the partonic structure of the proton within the QCD framework.

\section{NC $e^{ \pm} p$ cross section at high Bjorken $x$}

Neutral current $e^{ \pm} p$ DIS double-differential cross sections as a function of $x$ and $Q^{2}$ for $Q^{2}>$ $725 \mathrm{GeV}^{2}$ and up to $x \cong 1$ [1]. An improved reconstruction method and increased amount of data allows to achieve a high precision of the measurement. The obtained cross sections show a good agreement with the predictions from the Standard Model (SM). The comparison of the measured $e^{+} p$ cross sections and SM expectations based on the HERAPDF 1.5 set of PDFs [2] is presented in Figure 1 .

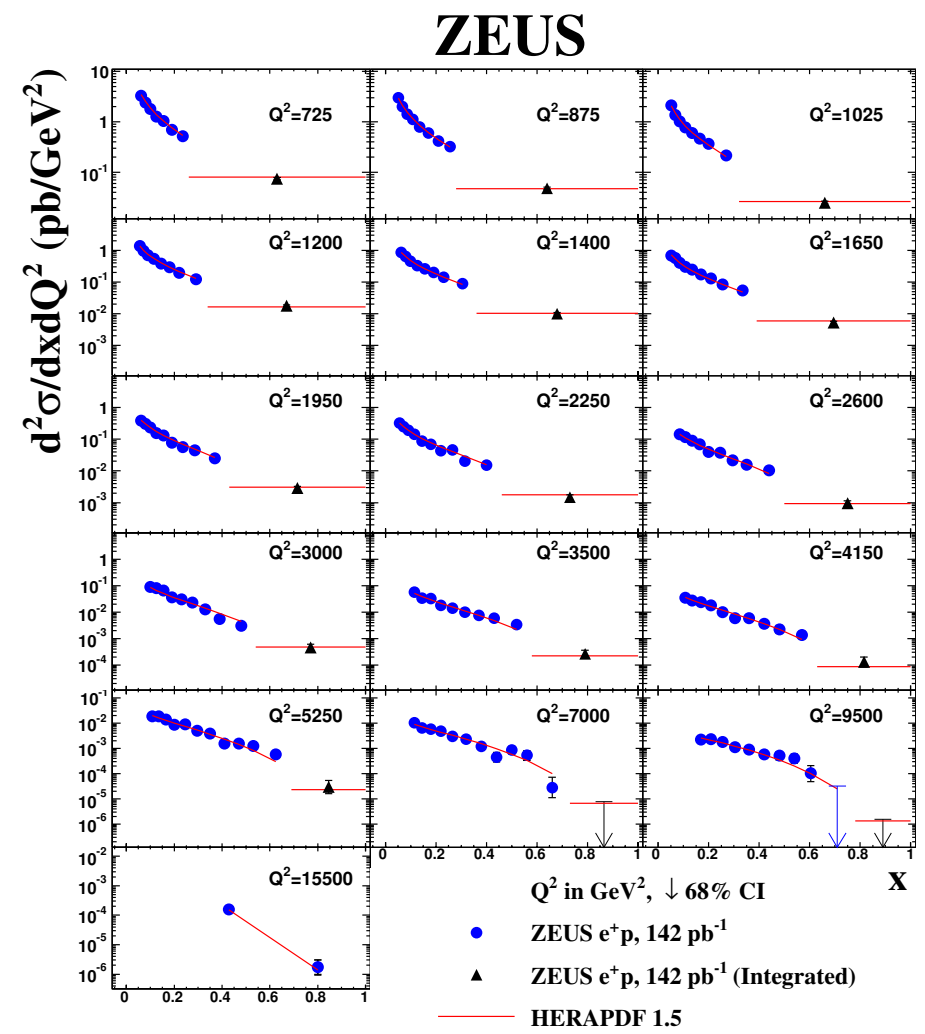

Figure 1. The double-differential cross section for $\mathrm{NC} e^{+} p$ scattering at $\sqrt{s}=318 \mathrm{GeV}$ (dots) as a function of $x$ and the double differential cross section integrated over $x$ divided by the bin width and placed at the centre of the bin (triangles) for different values of $Q^{2}$ as shown, compared to the Standard Model expectations evaluated using HERAPDF1.5 PDFs (line). The error bars show the statistical and systematic uncertainties added in quadrature. For the bins with zero measured events, a $68 \%$ probability limit is given.

\section{DIS cross section at different centre-of-mass energies and $F_{L}$}

The inclusive NC DIS cross sections for ep interactions are measured by the H1 experiment at two centre-of-mass energies of $\sqrt{s}=225$ and $251 \mathrm{GeV}$ in the region $35 \leq Q^{2} \geq 800 \mathrm{GeV}^{2}$ and up to the highest accessible inelasticity of $y=0.85$ [3]. The data are used together with previous measurement at $\sqrt{s}=318 \mathrm{GeV}$ to simultaneously extract the $F_{L}$ and $F_{2}$ structure functions. The ratio $R$ of the longitudinally to transversely polarised virtual photon cross section is consistent with being constant 
over the kinematic range of the data and is determined to be $0.23 \pm 0.04$. The $F_{L}$ measurements are used to perform a gluon density extraction based on a NLO approximation which is found to have a reasonable agreement with the gluon determined from the scaling violations.

A new measurement of the reduced $e^{+} p$ cross sections up to large values of $y$ is performed by the ZEUS expariment [4]. The analysed data is taken at different centre-of-mass energies, $\sqrt{s}=318,251$ and $225 \mathrm{GeV}$, allowing for measurements at fixed $x$ and $Q^{2}$ but different $y$ values. This allows to decouple $F_{2}$ and $F_{l}$, providing direct sensitivity to the gluon density. The measurement is performed in the kinematic region $0.13 \leq y \geq 0.75$ and $5 \leq Q^{2} \geq 110 \mathrm{GeV}^{2}$. The ration $R$ is also determined as a function of $Q^{2}$ together with an overall value of $R=0.105_{-0.037}^{+0.055}$.

The $F_{L}$ values extracted by the $\mathrm{H} 1$ are compared to the results of the similar analysis by ZEUS and NNLO QCD predictions based on HERAPDF1.5 set of PDFs in in Figure 2. The same figure contains the comparison of $R$ ratio of the longitudinally to transversely polarised virtual photon cross section obtained by each of the experiments.

\section{ZEUS}
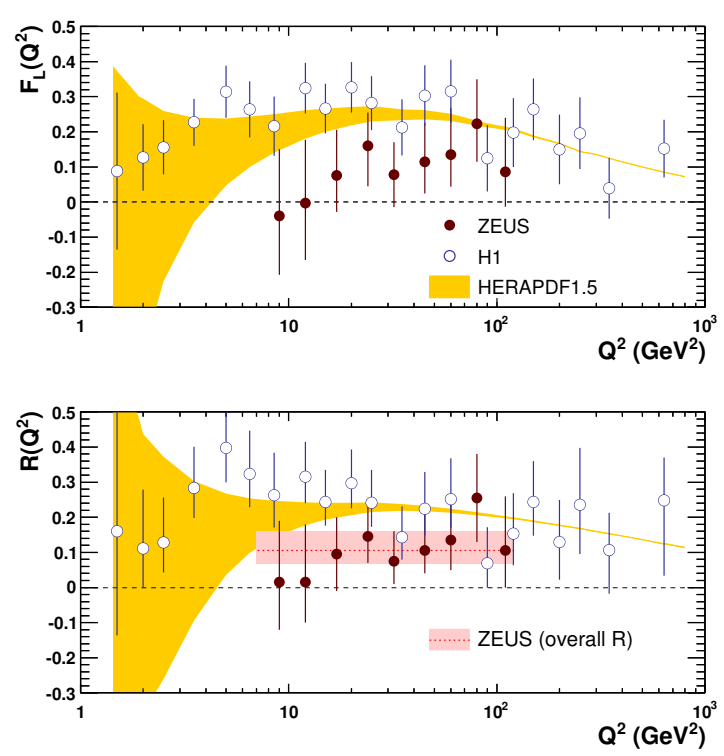

Figure 2. Values of (upper plot) $F_{L}$ and (lower plot) $R$ as a function of $Q^{2}$. The ZEUS data are shown as filled circles, with error bars representing the combined statistical and systematic uncertainties. The values of $F_{L}$ at different $Q^{2}$ points are correlated, as well as the values of $R$. The shaded band labelled "ZEUS (overall $R$ )" represents the $68 \%$ probability interval for the overall $R$. The $\mathrm{H} 1$ data are shown as open circles with error bars representing the total uncertainties. The ZEUS and H1 points are measured at somewhat different $x$ values. NNLO QCD predictions from HERAPDF1.5 are also shown. The bands indicate the uncertainty in the predictions.

\section{Combination and QCD analysis of the inclusive $e^{ \pm} p$ cross sections measured at HERA}

All of the inclusive DIS cross sections measured by both H1 and ZEUS collaborations in NC and CC unpolarised $e^{ \pm} p$ scattering at HERA [5]. The data correspond to a luminosity of about $1 \mathrm{fb}^{-1}$ and span six orders of magnitude in negative four-momentum-transfer squared, $Q^{2}$, and Bjorken $x$. They include data taken at proton beam energies of $920,820,575$ and $460 \mathrm{GeV}$. The combination is performed using the packages HERAverager and HERAFitter [6, 7]. The correlated systematic uncertainties and global normalisations are averaged such that one coherent data set is obtained. Since H1 and ZEUS have employed different experimental techniques, using different detectors and methods 


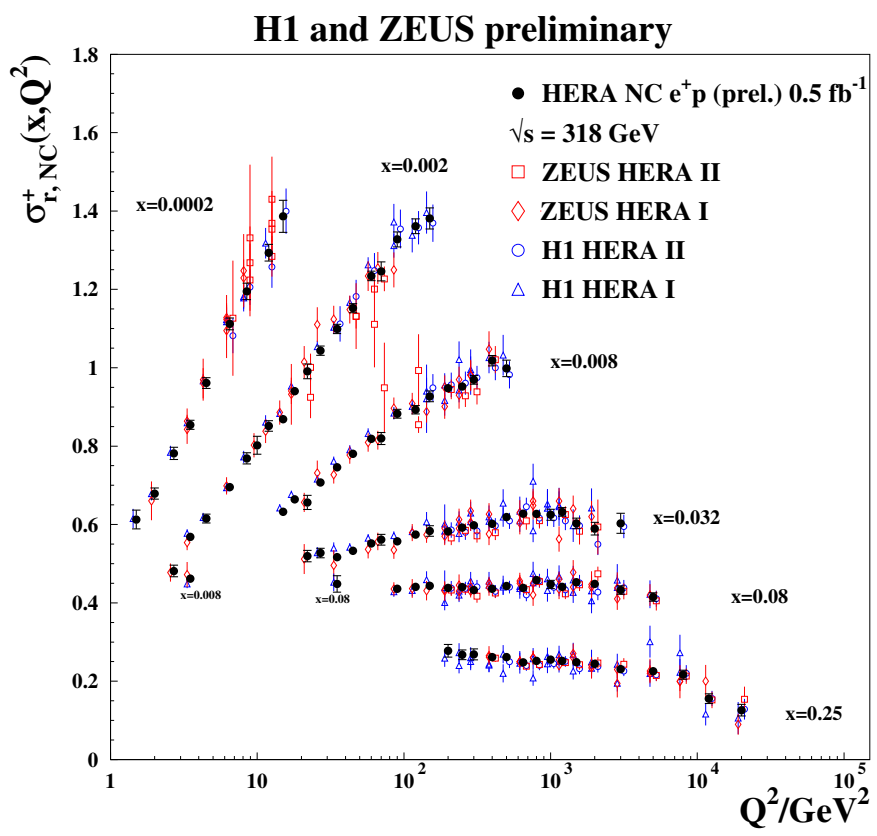

Figure 3. HERA combined $\mathrm{NC} e^{+} p$ reduced cross section as a function of $Q^{2}$ for six selected $x$-bins compared to the separate $\mathrm{H} 1$ and ZEUS data which were the input to the averaging procedure. The individual measurements are displaced horizontally for better visibility. Errors bars represent the total uncertainties

of kinematic reconstruction, the combination leads to a significantly reduced uncertainty. Figure 3 shows the averaged $\mathrm{NC} e^{+} p$ reduced cross sections together with the input data from $\mathrm{H} 1$ and ZEUS for $e^{+} p$ scattering.

The newly combined HERA I and II data are input to QCD analyses at NLO and NNLO, which determine preliminary versions of new sets of parton distributions, HERAPDF2.0(prel.) [8]. The QCD predictions for the structure functions are obtained by solving the DGLAP evolution equations. at NLO and NNLO in the $\overline{M S}$ scheme with the renormalisation and factorisation scales chosen to be $Q^{2}$. The program QCDNUM [7] is used within the HERAFitter framework. The DGALP equations yield the PDFs at all values of $Q^{2}$ if they are provided as functions of Bjorken $x$ at some input scale $Q_{0}^{2}$. The light quark coefficient functions are calculated in QCDNUM, while the heavy quark coefficient functions are calculated in the general mass variable flavour scheme RTOPT [9, 10]. Figure 4 shows summary plots of the HERAPDF2.0(prel.) at NLO and NNLO.

\section{Multijet production at HERA and the strong coupling constant $\alpha_{s}$}

Inclusive jet, dijet and trijet differential cross sections are measured in NC DIS for exchange boson virtualities $150<Q^{2}<15000 \mathrm{GeV}^{2}$ using the H1 detector at HERA [11]. The data were taken in the years 2003 to 2007 and correspond to integrated luminosity of $351 \mathrm{pb}^{-1}$. Double differential jet cross sections are obtained using a regularised unfolding procedure to correct for detector effects. The measurement is done as a function of $Q^{2}$ and the transverse momentum of the jet $P_{T}^{\text {jet }}$, and as a function of $Q^{2}$ and the proton's longitudinal momentum fraction, $\xi$, carried by the parton participating in the hard interaction. In addition normalised double differential jet cross sections are measured as the ratio of the jet cross sections to the inclusive NC cross sections in the respective $Q^{2}$ bins of the jet measurements, which benefits from cancellation of systematic uncertainties correlated between the measurements. Figure 5 shows the measured cross sections as a function of $Q^{2}$ and $P_{T}$. 

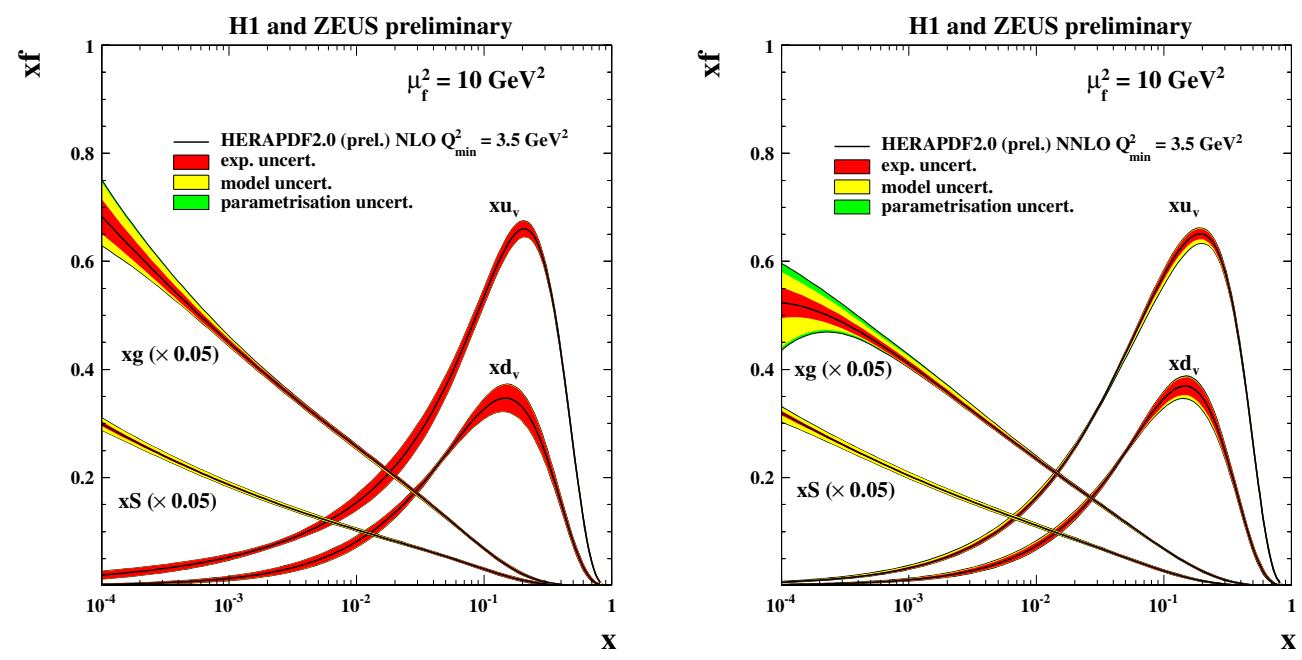

Figure 4. The parton distribution functions from HERAPDF2.0(prel.) at NLO (left) and NNLO (right) at the scale $\mu_{f}^{2}=10 \mathrm{GeV}^{2}$. The gluon and sea distributions are scaled by a factor 20 . The experimental, model and parametrisation uncertainties are shown separately.

The measurements of the inclusive, the dijet and the trijet cross section are used separately and also simultaneously to extract values for the strong coupling constant $\alpha_{s}\left(M_{Z}\right)$. The best experimental precision of $0.7 \%$ is obtained when using the normalised multijet cross sections. The simultaneous extraction of the strong coupling constant $\alpha_{s}\left(M_{Z}\right)$ from the normalised inclusive jet, the dijet and trijet samples yields: $\alpha_{s}\left(M_{Z}\right)=0.1165 \pm 0.0008_{\text {exp }} \pm 0.0038_{\text {theo }}$.

The running of the $\alpha_{s}$, determined from the normalised cross section, is shown to be consistent with the expectation from the renormalisation group equation and with values of $\alpha_{s}$ from other jet measurements. The comparison is shown in Figure 6.

\section{References}

[1] H. Abramowicz et al. (ZEUS Collaboration), Phys. Rev. D 89, 072007 (2014)

[2] V. Radescu (H1 and ZEUS Collaborations) (2013), 1308.0374

[3] V. Andreev et al. (H1 Collaboration), Eur.Phys.J. C74, 2814 (2014), 1312.4821

[4] H. Abramowicz et al. (ZEUS Collaboration) (2014), 1404.6376

[5] H1 and ZEUS Collaborations (2014), H1prelim-14-041, ZEUS-prel-14-005

[6] F. Aaron et al. (H1 and ZEUS Collaboration), JHEP 1001, 109 (2010), 0911.0884

[7] M. Botje, Comput.Phys.Commun. 182, 490 (2011), 1005 . 1481

[8] H1 and ZEUS Collaborations (2014), H1prelim-14-042, ZEUS-prel-14-007

[9] R. Thorne, R. Roberts, Phys.Rev. D57, 6871 (1998), hep-ph/9709442

[10] R. Thorne, Phys.Rev. D73, 054019 (2006), hep-ph/0601245

[11] V. Andreev et al. (H1 Collaboration) (2014), 1406.4709 


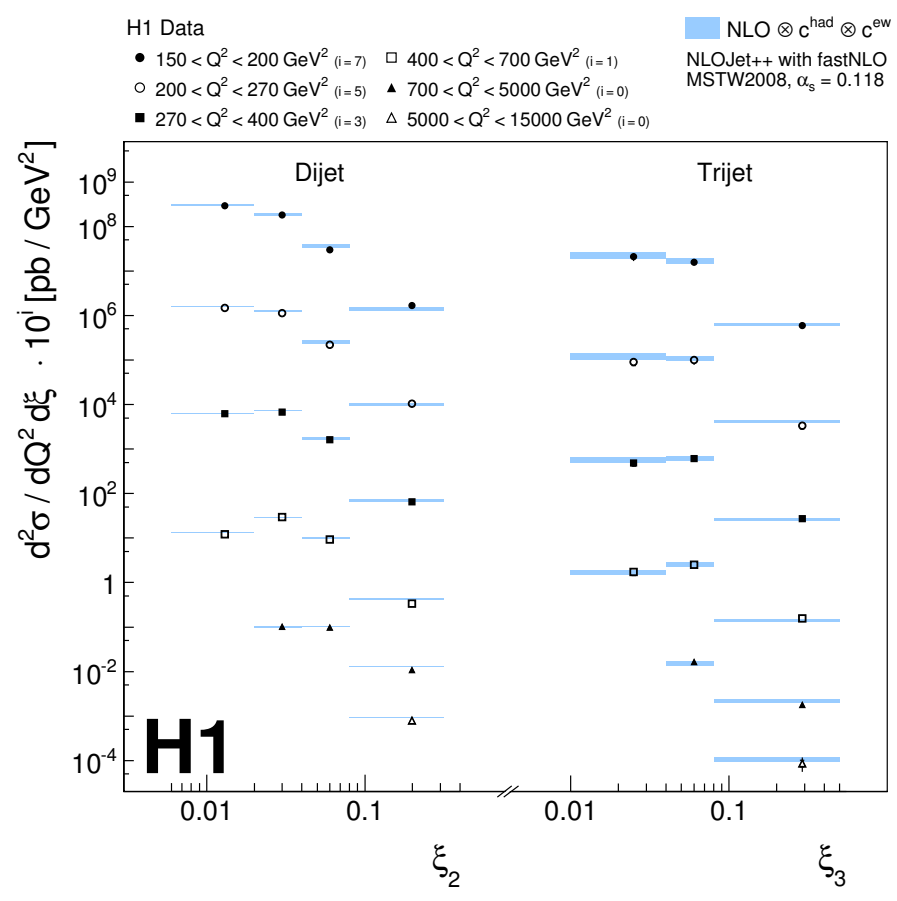

Figure 5. Double differential cross sections for jet production in DIS as a function of $Q^{2}$ and $P_{T}$. The inner and outer error bars indicate the statistical uncertainties and the statistical and systematic uncertainties added in quadrature. The NLO QCD predictions, corrected for hadronisation and electroweak effects, together with their uncertainties are shown by the shaded band. The cross sections for individual $Q^{2}$ bins are multiplied by a factor of $10^{i}$ for better readability. 


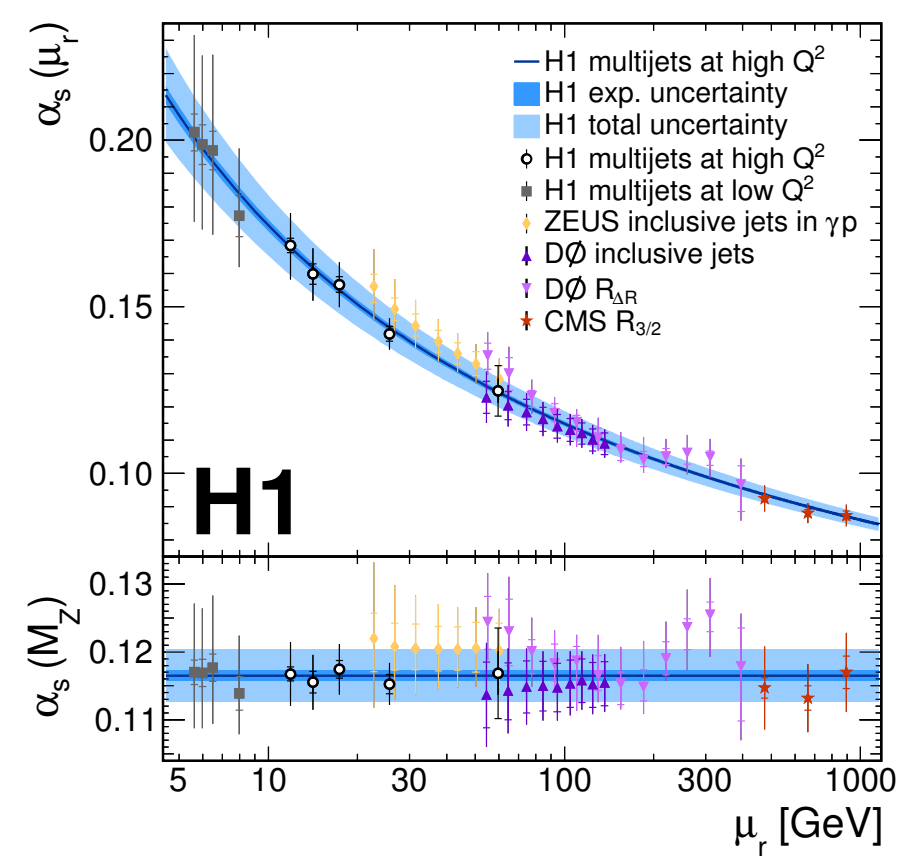

Figure 6. The upper panel shows the values of the strong coupling $\alpha_{s}\left(\mu_{r}\right)$ as determined from the normalized multijet measurement (open dots) at different scales $\mu_{R}$. The solid line shows the NLO QCD prediction calculated using the renormalisation group equation with $\alpha_{s}=0.1165$ as determined from the simultaneous fit to all normalized multijet measurements. The dark shaded band around this line indicates the experimental uncertainty on $\alpha_{s}\left(\mu_{r}\right)$, while the light shaded band shows the total uncertainty. Also shown are the values of from multijet measurement at low values of $Q^{2}$ by H1 (squares), from inclusive jet measurements in photoproduction by the ZEUS experiment (diamonds), from in clusive jet measurement and jet angular correlations $R_{\Delta R}$ by the $\mathrm{D} 0$ experiment at the Tevatron (upper and lower triangles), and from the ratio of trijet to dijet cross sections as measured by the CMS experiment at the LHC (stars). In the lower panel the equivalent values of $\alpha_{s}\left(M_{Z}\right)$ for all measurements are shown. 
